\title{
Minimal Hepatic Encephalopathy is an under Recognized Entity in Clinical Practice of Bangladeshi Physician
}

\author{
MF ABEDIN ${ }^{\mathrm{a}}$, MJ ABEDIN $^{\mathrm{b}}$, MA MAHTAB $^{\mathrm{c}}, \mathrm{N}^{\mathrm{AHMAD}}{ }^{\mathrm{d}}$
}

\begin{abstract}
:
Background: Minimal Hepatic Encephalopathy, the mildest from of Hepatic Encephalopathy is characterized by subtle motor and cognitive deficits and impairs health related quality of life. Though the prevalence of Minimal Hepatic Encephalopathy in cirrhotic patient is high but awareness regarding MHE is yet not satisfactory. Moreover diagnosis of MHE, the cut off normative value for psychometric test is yet not established in Bangladesh. This is the first study in Bangladesh to find out the normative value for psychometric test and see the prevalence of Minimal Hepatic Encephalopathy in cirrhotic patient.

Methods: Cross sectional study done in Department of Hepatology, BSMMU, Dhaka from July 2012 to June 2014. Total 150 patient of which 50 patient with cirrhosis and remaining 100 healthy individual were included in the study. By doing number connection test, Serial dotting test and line tracing test in healthy individual, first normative values for psychometric test was detected then these test was done on cirrhotic patient, whose 2 psychometric test result among 3 above normal value were enrolled as a case of Minimal Hepatic Encephalopathy.
\end{abstract}

\section{Background}

Hepatic Encephalopathy (HE) is a neuropsychiatric syndrome in patients with liver disease and/or portosystemic shunting, which symptoms may vary from

a. Dr. Md. Forhad Abedin, MBBS, FCPS(Int.Med), MD (hepatology), Resident Physician, Comilla Medical College Hospital, Comilla, Bangladesh

b. Dr. Md. Joynal Abedin, MBBS, BCS (Health), Assistant Registrar, Department of Medicine, Dinajpur Medical College Hospital, Dinajpur, Bangladesh.

c. Dr. Mamun Al Mahtab, Associate Professor, Department of Hepatology, Bangabandhu Sheikh Mujib Medical University, Dhaka, Bangladesh.

d. Prof. Nooruddin Ahmad, Professor, Department of Hepatology, Bangabandhu Sheikh Mujib Medical University, Dhaka, Bangladesh.

Address of Correspondence: Dr. Mohammed Forhad Abedin, MBBS, FCPS(Medicine), MD(hepatology), Resident Physician,Comilla Medical College Hospital, Comilla, Bangladesh. Contacts: Email:fabedin75@gmail.com. Mobile:+8801716741473

Received: 5 June 2017

Accepted: 25 Feb. 2018
None of the patient previously diagnosed as any type of Hepatic Encephalopathy.

Results: Cut off normative value for NCT, SDT, LTT is 52 seconds, 52 seconds and 84 seconds respectively (Mean+2SD). Prevalence of Minimal Hepatic Encephalopathy in this study was $66 \%$ and it is more prevalent in advanced cirrhosis.

Conclusion: MHE is frequent in patient with liver cirrhosis, manifested even in patient with child pugh A liver cirrhosis. Every attention should be given to detect Minimal Hepatic Encephalopathy in patient with cirrhosis of liver well before the development of overt Hepatic Encephalopathy.

Key Word: Number connection test (NCT), Serial dotting test (SDT), line tracing test (LTT).

Abbreviation Used: MHE: Minimal Hepatic Encephalopathy, HE: Hepatic Encephalopathy EEG: Electroencephalography, CP: Child Pugh, LFT: Liver function test.

(J Bangladesh Coll Phys Surg 2018; 36: 59-63) DOI: http://dx.doi.org/10.3329/jbcps.v36i2.36067

subtle memory or attention deficits to deep coma ${ }^{1}$. The aetiology of $\mathrm{HE}$ is a diminished hepatic clearance of toxins of intestinal origin in case of liver insufficiency and/or by the hepatic bypassing of these toxins in case of portosystemic shunting1. These toxins thus bypass the liver and enters the systemic circulation, causing the primary and secondary changes in brain neurochemistry that produce symptoms of hepatic encephalopathy. This metabolic disorder is characterized by reversibility, which suggest a lack of persistent structural lesion in the brain ${ }^{1}$. HE may therefore not only occur in patients with acute as well as chronic liver disease but also in patients with a portosystemic shunt without liver disease. These different aetiological aspects of HE are reflected in a recently proposed nomenclature that divides this neuropsychiatric syndrome into types A (associated with acute liver failure), B (associated with portosystemic shunting or bypass) and $\mathrm{C}$ (associated with liver cirrhosis) ${ }^{2}$.

Minimal Hepatic Encephalopathy, the mildest form of hepatic encephalopathy is characterized by subtle 
motor and cognitive deficits and impairs health related quality of life ${ }^{3}$. This group of patient has normal mental and neurological status on standard clinical examination but exhibit a number of neuropsychiatric and neurophysiological defects ${ }^{4}$.

According to the recommendation of world congress of Gastroenterology, minimal hepatic encephalopathy is a better term because the word subclinical may be mistaken as signifying lack of clinical importance ${ }^{2}$. Minimal hepatic encephalopathy is present in $25 \%$ to $80 \%$ of cirrhotic patient without overt hepatic encephalopathy 5 . Although named "minimal", minimal hepatic encephalopathy can have a far reaching impact on quality of life and progression to overt hepatic encephalopathy ${ }^{6}$.

Cirrhotic patients with MHE more frequently develop episodes of overt HE than those without $\mathrm{MHE}^{4}$. Though the prevalence of minimal hepatic encephalopathy in cirrhotic patient is high but awareness regarding minimal hepatic encephalopathy is yet not satisfactory. This study is an attempt to evaluate the minimal hepatic encephalopathy in patient with cirrhosis of liver in an university hospital of Bangladesh.

\section{Methodology:}

It was a cross sectional, case control study done in Department of Hepatology, BSMMU, Dhaka from July 2012 to June 2014. Patient were selected by non probability convenience sampling from outpatient and inpatient department of hepatology, BSMMU, Dhaka. Total 150 patient of which 50 patient with cirrhosis as cases and remaining 100 healthy individual as controlled were included in the study. Cases were included having age greater than 20 years and less than 60 years, diagnosed to have cirrhosis of liver by history, clinical examination, laboratory findings and ultrasonography, not on medications causing cognitive defects like benzodiazepines and having normal mental status on clinical examination. Those patients were excluded who had overt hepatic encephalopathy, other psychiatric and neurological disease causing cognitive dysfunction, difficulties in performing psychometric tests such as those with bad vision, taking hepatotoxic drugs and patient with primary neoplasm and secondaries in liver recognized by Ultrasonography.

Control group were taken from age range between 20 to 60 years, capable of reading and writing, non alcoholics, do not have documented evidence of acute or chronic liver disease and not on medication causing cognitive defects like benzodiazepines. Prior to the commencement of the study, the research protocol was approved by the ethical institutional review board of BSMMU, Dhaka. The aims and objectives of the study along with its procedure, risk and benefits of the study was explained to the patient in easily understandable local language. Then informed consent was taken from each patient. Neuropsychological test was done firstly in control group. Hundred healthy people were selected according to inclusion criteria for control group. Then 3 psychometric test (number connection test, serial dotting test, line tracing test) was done on control group. Before doing the test, they were demonstrated about psychometric test. Paper \& ball point pen was given to the person who was tested \& observer was ready with stopwatch. When observer called start, person started the test. If there is any wrong by the person, observer corrected the procedure. Number needed to correct was not documented, but time taken by person was written in procedure sheet by the observer. These data was analyzed by SPSS 20 and from that calculation of normal cut off value for three psychometric test (by mean+2SD)was done. Then the psychometric test was done on cases for the diagnosis of minimal hepatic encephalopathy. Cirrhotic patient who were positive for two psychometric test among three, were enrolled as a case of minimal hepatic encephalopathy.

\section{Results:}

Among one hundred and fifty patient, 50 patients were diagnosed as a case of cirrhosis of liver by standard clinical biochemical and radiological examination and remaining100 patients were taken as control for the neuropsychiatric test. None of the patient had evidence of neurological and/or psychiatric abnormalities on global clinical examination. The demographic and clinical characteristics of the patient are summarized in table-I.

The case group comprise of 50 patient with mean age of 41.36 years, minimum age of 20 years and maximum of 60 years. In the control group of 100 cases the mean age was 27.65 years, minimum age of 20 years and ,maximum of 60 years. Among 50 cases, 35 were diagnosed by hepatologist 10 cases were 
diagnosed by gastroenterologist and 5 by internal medicine specialist and other specialist. None of those cases were tasted for minimal hepatic encephalopathy by any psychometric tests before as shown in table-II.

In the control group of 100 cases the mean value for number connection test was $34.85 \pm 9.07$, mean value for serial dotting test was $40.38 \pm 6.8$ seconds, mean value for line tracing test was $54.43 \pm 15.59$ seconds. So normal cut off value for three psychometric test (by mean+2SD) was 52 seconds for Number Connection Test, 52 seconds for Serial Dotting Test and 84 seconds for Line Tracing Test. Values of three psychometric test among case and control with their $\mathrm{p}$ values are shown in table-III

In fifty cases mean value of number connection test was 75 seconds when compared to control it was 34 seconds. Among the cases 39(78\%) patient scored above the cut off point of controls which was statistically significant

Table-I

Characteristics of the studied patients

Patient $(\mathrm{n}=50)$

\begin{tabular}{lc}
\hline Mean age, years(Range) & $41.36 \pm 10.43(20-60)$ \\
Gender (Male/Female) & $44 / 6$ \\
Inpatient /Out patient & $22 / 28$ \\
Etiology of cirrhosis & \\
$\quad$ HBV & 41 \\
$\quad$ HCV & 4 \\
$\quad$ Cryptogenic & 4 \\
Alcoholic & 1 \\
Child Pugh Class $(\mathrm{A} / \mathrm{B} / \mathrm{C})$ & $16 / 24 / 10$ \\
Esophageal Varices $($ Grade $1 / 2 / 3)$ & $16 / 17 / 13$ \\
Blood Amonia Level $(\mu \mathrm{mol} / \mathrm{L})$ & $44.32 \pm 23.17$ \\
\hline
\end{tabular}

Table-II

Number of cases seen by specialists and their previous psychometric analysis status

\begin{tabular}{lcc} 
Specialist & number of cases & previous psychometric test \\
\hline Hepatologist & 35 & Not done \\
Gastroenterologist & 10 & Not done \\
Internal Medicine Specialist and other & 5 & Not done \\
\hline
\end{tabular}

Table-III

Values of three psychometric test among case and control with their $p$ value

\begin{tabular}{lcccccc} 
Test & Group & $\mathrm{N}(\mathrm{n})$ & $\begin{array}{c}\text { Mean } \\
\text { (Seconds) }\end{array}$ & SD & $\begin{array}{c}\text { Cut off value } \\
\text { (Mean+2SD) } \\
\text { Seconds }\end{array}$ \\
\hline Number Connection Test & Control & 100 & 34 & 9 & 52 & $<0.001$ \\
& Case(n) & 50 & 75 & 25 & 52 & $<0.001$ \\
Serial Dotting Test & Control & 100 & 40 & 6 & 21 & 84 \\
Line Tracing Test & Case(n) & 50 & 71 & 15 & & $<0.001$ \\
\hline
\end{tabular}

61 
with a $\mathrm{p}$ value $<0.001$. The mean value of SDT was 71 seconds when compared to control it was 40 seconds. Among the cases $36(72 \%)$ patient scored above the cut off value of control which was statistically significant with a $\mathrm{p}$ value $<0.001$. Mean value of line tracing test was 96 seconds when compared to control it was 54 seconds. Among the cases 31(62\%) scored above the cut off point of control which was statistically significant with a $\mathrm{p}$ value $<0.001$.

Among 50 cases, 33 scored beyond the cut off point of at least 2 psychometric test. So in this study the prevalence of minimal hepatic encephalopathy is about $66 \%$. Frequency of psychometric test result among cases shown in figure-I. Prevalence of minimal hepatic encephalopathy increase in advanced cirrhosis. It is about $72.72 \%$ in Child Pugh B \& C. Whereas in Child Pugh A it is $27 \%$.It is shown in figure-II.

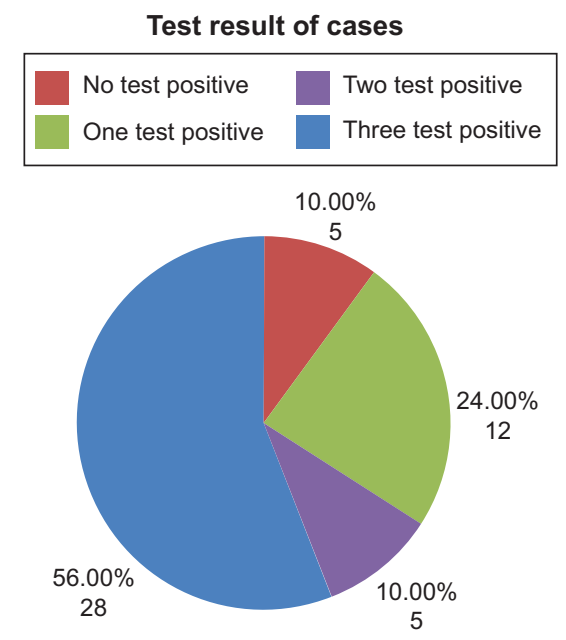

Fig.-1: Frequency of psychometric test result among cases.

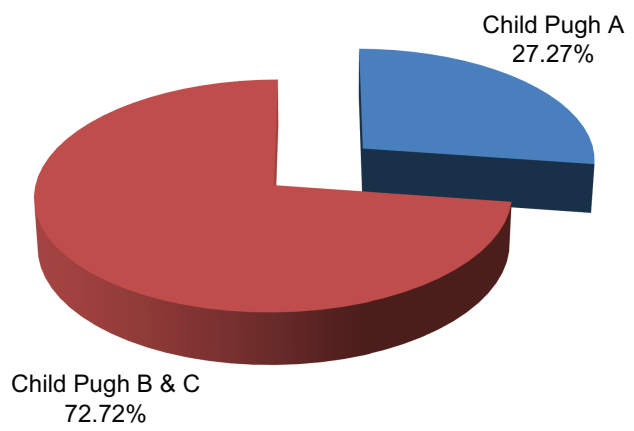

Fig.-2: prevalence of Minimal Hepatic Encephalopathy in different Child Pugh Classes.

\section{Discussion:}

The diagnosis of minimal hepatic encephalopathy is based on a careful neuropsychiatric evaluation. No single test is diagnostic for minimal hepatic encephalopathy. A standardized test battery including NCT A \& B, The Line Tracing Test, Serial Dotting Test and the Digit Symbol Test is recommended.

However in one study it is shown that diagnosis of minimal hepatic encephalopathy can be done $96 \%$ sensitivity and $100 \%$ specificity $^{7}$, if at least 2 of the above mentioned test is beyond their cut off value . The sensitivity of the EEG and blood Ammonia level for the diagnosis of MHE is limited. In our study we have assessed 50 cases of cirrhosis patient by using Number Connection Test, Serial Dotting Test and Line Tracing Test. We also established a cut off value for this test by doing the test in 100 healthy individuals.

The mean age in the present study was 41.36 years for case group and 27.56 years for the control groups. The majority of our patient were males. They constitute for about $88 \%$ and $86 \%$ in cases and control group respectively. However in the previous study done by quero and others ${ }^{8}$ the mean age was 49 years with range from 27 to 77 years. Mean age in the study of 179 patients by Groenweg and his colleague ${ }^{9}$ was 50 years with 113 males and 66 females.

Prevalence of minimal hepatic encephalopathy among cirrhotic patient ranges from $20 \%$ to $84 \%{ }^{4}$. In our study the prevalence of minimal hepatic encephalopathy is $66 \%$ which is concordant with previous studies ${ }^{8}$. Some author consider it as an epidemic pathology ${ }^{10}$. This wide range in minimal hepatic encephalopathy prevalence is because of difference in definition, lack of standardized diagnostic criteria, difference in diagnostic method, the clinico pathological co morbid spectrum and socio demographic variables.

Like many other factors advanced liver disease is also responsible for the increase prevalence of minimal hepatic encephalopathy. In different study it was shown that prevalence of minimal hepatic encephalopathy is less than 15\% in Child Pugh A class and it is more than $50 \%$ in Child Pugh B//C class ${ }^{11}$. In our study prevalence MHE in Child Pugh A class is $27 \%$ and it was about $72 \%$ in Child Pugh B \& C which is concordant with other studies. 
MHE may affect multiple aspect of brain function such as perception, memory, attention, mental speed ${ }^{12}$ etc. Neuropsychological test (NCT, SDT, LTT) designed to recognize those brain dysfunction ${ }^{13}$. Normal Cut off point of this paper pencil test obtained first from healthy control. There are available normal cut off value for German Italian and Spanish population which is significantly different from each other ${ }^{14}$. So it is utmost important to set a normal cut off value of those psychometric test before using it as tools for diagnosis of minimal hepatic encephalopathy in our context. In this study normal value for Number Connection Test up to 52 seconds, Serial Dotting Test is up to 52 seconds and Line Tracing Test is up to 84 seconds.

\section{Conclusion}

Minimal Hepatic Encephalopathy is frequent in patient with liver cirrhosis manifested even in patient with Child A liver function. Its severity increase as liver function deteriorates, being most severe with Child C liver function. The wide prevalence of minimal hepatic encephalopathy is likely to impact adversely on the quality of life of cirrhotic patient. So every attention should be given to detect Minimal Hepatic Encephalopathy in patient with cirrhosis of liver who yet not manifested as a case of overt hepatic encephalopathy.

\section{Conflict of Interest}

\section{The authors have none to declare}

\section{References:}

1 Souheil A $Z$ et al. Metabolic consequences of cirrhosis often is reversible. Post graduate Medicine 2001;109(2):521-6.

2 Ferenci $\mathrm{P}$ et al. Hepatic encephalopathy -definition , nomenclature, diagnosis and quantification: final report of the working party at the $11^{\text {th }}$ world congress of Gastroenterology, Vienna, 1998, Hepatology. 2002 Mar;35(3):716-21

3 Das A et al. Prevalence and natural history of subclinical hepatic encephalopathy in cirrhosis. J Gastroenterol hepatol 2001; 16:531-35.

4 Praveen Sharma Minimal hepatic encephalopathy JAPI November 20009-Vol-57.

5 Dhiman et al. figure connection test : A universal test for assessment of mental state. J Gastroenterol Hepatol 10:1423,1995 .

6 Hartman I J et al. the prognostic significance of subclinical hepatic encephalopathy. Am J Gastroenterol 2000; 95:2029-34.

7 Luis Eduardo, Zamora Nava, Aldo Torre Delgadillo. Minimal hepatic encephalopathy. Annals of Hepatology 2001;10 suppl 2:50-55.

8 Quero J C et al, The diagnosis of subclinical hepatic encephalopathy in patient with cirrhosis using neuropsychological test and automated electro-encephalogram analusis. Hepatology 1996;24:556-60.

9 Groeneweg et al. Screening of Subclinical hepatic encephalopathy. J heaptol 2000; 32: 748-753

10 Bajaj J et al. Minimal hepatic encephalopathy matters in daily life. World J Gastroenterol 2008;14:3609-15.

11 Kircheis $\mathrm{G}$ et al. Critical Flicker frequency for quantification of low grade hepatic encephalopathy. Hepatology 2002; 35: $357-66$.

12 Cordoba J, Cabrera J, Lataif L, Penev P, Zee P, Blei AT. High prevalence of sleep disturbance in cirrhosis. Hepatology 1998;27:339-345.

13 Weissenborn K, Ennen JC, Ru "ckert N, Hecker H. Neuropsychological characterization of hepatic encephalopathy. J Hepatol 2001;34: 768-773.

14 Randolph et al. Neuropsychological assessment of hepatic encephalopathy: ISHEN practice guidelines. Liver International 2009;29(5):629-35. 